\title{
Vibration-Driven Self-Doping of Dangling-Bond Wires on Si(553)-Au Surfaces
}

\author{
C. Braun $\odot,{ }^{1, *}$ S. Neufeld, ${ }^{1}$ U. Gerstmann, ${ }^{1}$ S. Sanna, ${ }^{2}$ J. Plaickner $\odot,{ }^{3}$ E. Speiser, ${ }^{3}$ N. Esser, ${ }^{3,4}$ and W. G. Schmidt ${ }^{1}$ \\ ${ }^{1}$ Lehrstuhl für Theoretische Materialphysik, Universität Paderborn, 33095 Paderborn, Germany \\ ${ }^{2}$ Institut für Theoretische Physik and Center for Materials Research, Justus-Liebig-Universität Gießen, 35392 Gießen, Germany \\ ${ }^{3}$ Leibniz-Institut für Analytische Wissenschaften -ISAS- e.V. Schwarzschildstr. 8, 12489 Berlin, Germany \\ ${ }^{4}$ Technische Universität Berlin, Institut für Festkörperphysik, Hardenbergstr. 36, 10623 Berlin, Germany
}

(Received 16 October 2019; accepted 21 February 2020; published 7 April 2020)

\begin{abstract}
Density-functional theory is used to explore the $\mathrm{Si}(553)$-Au surface dynamics. Our study (i) reveals a complex two-stage order-disorder phase transition where with rising temperature first the $\times 3$ order along the Si step edges and, subsequently, the $\times 2$ order of the Au chains is lost, (ii) identifies the transient modification of the electron chemical potential during soft Au chain vibrations as instrumental for disorder at the step edge, and (iii) shows that the transition leads to a self-doping of the Si dangling-bond wire at the step edge. The calculations are corroborated by Raman measurements of surface phonon modes and explain previous electron diffraction, scanning tunneling microscopy, and surface transport data.
\end{abstract}

DOI: 10.1103/PhysRevLett.124.146802

Dangling-bond $(\mathrm{db})$ nanostructures are potential fundamental building blocks for atom-scale electronics [1-4]. Given the electronic instabilities in one-dimensional systems [5-8], it is a particular challenge to render db wires metallic and much effort is spent to tune their conductance [9-13] and magnetic properties [14,15].

The Si(553)-Au surface, providing self-organized $\mathrm{Si} \mathrm{db}$ wires [16-18], is a prototype example. It consists of $\mathrm{Si}(111)$ terraces separated by single atomic steps and is stabilized by a submonolayer of Au atoms; see Fig. 1. The gold atoms form a double chain on the terrace, next to a Si honeycomb chain at the step edge [19]. The Si step edge atoms feature dangling bonds (dbs). Two fully occupied $s p^{3}$ hybridized $\mathrm{dbs}$ alternate with one empty $p$ orbital of $s p^{2}$ hybridized $\mathrm{Si}$ in the ground state $(2,2,0)$ electron configuration [20]. This $\times 3$ periodicity together with $\mathrm{a} \times 2$ periodicity due to $\mathrm{Au}$ chain dimerization is seen by scanning tunneling microscopy (STM) [17,21-23] and low-energy electron diffraction (LEED) [24]. Thermal excitation leads to a sudden conductivity increase along the step edge at around $65 \mathrm{~K}$. Further temperature increase quenches first the $\times 3$, and, subsequently, the $\times 2$ periodicity [25].

In this Letter, density-functional theory (DFT) calculations and Raman measurements are presented which (i) provide a consistent explanation for the experimental data and (ii) reveal a complex phase transition mechanism that involves the electron doping of the step edge $\mathrm{db}$ wire:

Published by the American Physical Society under the terms of the Creative Commons Attribution 4.0 International license. Further distribution of this work must maintain attribution to the author(s) and the published article's title, journal citation, and DOI.
A soft Au chain phonon mode is identified that facilitatesvia $\mathrm{Au} \rightarrow \mathrm{Si} \mathrm{db}$ charge transfer-an order-disorder transition at the Si step edge starting below $100 \mathrm{~K}$. At higher temperatures, the $\mathrm{Au}$ chain order is quenched.

In detail, DFT calculations were performed using the Vienna $a b$ initio simulation package (VASP) [26] and the PBEsol functional [27]. Collinear spin polarization is assumed. This methodology was recently shown to correctly describe the ground state of the $1 \times 6$ reconstructed $\mathrm{Si}(553)-\mathrm{Au}$ surface [20]. A supercell containing six $\mathrm{Si}$ bilayers (H passivated at the bottom), a $450 \mathrm{eV}$ plane-wave energy cutoff, and a $2 \times 3 \times 1$ Brillouin-zone sampling ensure numerical convergence. The Nosé-Hoover thermostat was used for $a b$ initio molecular dynamics (AIMD) calculations.

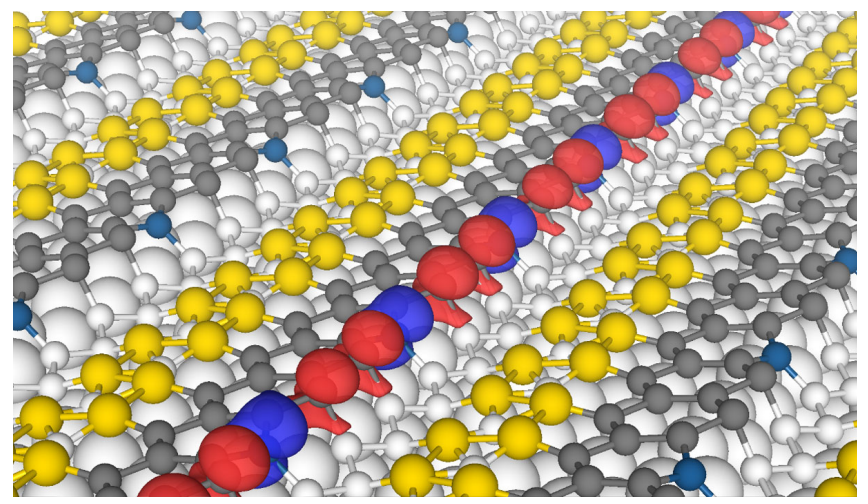

FIG. 1. Atomic structure of the $1 \times 6$ reconstructed $\mathrm{Si}(553)-\mathrm{Au}$ surface. Yellow, (dark) gray, and blue balls indicate Au, (honeycomb) $\mathrm{Si}$, and $\mathrm{Si}$ atoms with empty dangling bonds, respectively. Filled and empty dangling bonds in the ground state $(2,2,0)$ configuration are shown (only at one step edge) in red and blue, respectively. 


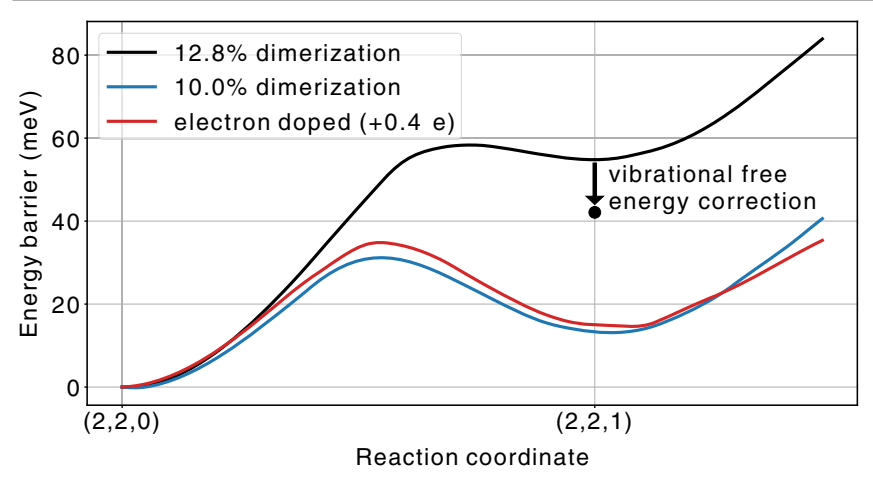

FIG. 2. Calculated potential energy surface from the $(2,2,0)$ ground state to the self-doped $(2,2,1)$ configuration for the AIMD calculated average dimerization at $100 \mathrm{~K}$ (12.8\%, black), a reduced dimerization $(10 \%$, blue) and a 0.4 electron doped $1 \times 6$ supercell (red). The dimerization is defined as $d=|a-b| / a$, where $a$ and $b$ are the Si lattice constant and $\mathrm{Au}-\mathrm{Au}$ bond length in chain direction, respectively. The reduction of the total energy difference between $(2,2,0)$ and $(2,2,1)$ structures due to vibrational free-energy corrections at $100 \mathrm{~K}$ is indicated.

Surface Raman measurements were performed in backscattering geometry using a triple Dilor-xy spectrometer with a high-efficiency Si-based CCD detector from Andor iDus. A $\mathrm{Kr}^{+}$laser operated at $647 \mathrm{~nm}$ is used as light source. This setup allows for the registration of Raman intensity down to $15 \mathrm{~cm}^{-1}$ with a spectral resolution below $3 \mathrm{~cm}^{-1}$. The sample temperature was controlled with a PID control loop, combining the operation of a closed-cycle $\mathrm{He}$ cryostat with a heating wire system.

Tegenkamp and co-authors [25] attribute the surface conductivity increase above $65 \mathrm{~K}$ to an $\mathrm{Au} \rightarrow \mathrm{Si}$ db charge transfer. Such a charge redistribution characterizes the spin-liquid structure suggested by Erwin et al. [14,24] for $\mathrm{Si}(553)-\mathrm{Au}$ : It has a $(2,2,1)$ electron configuration, i.e., two fully occupied dbs alternate with one singly occupied $\mathrm{db}$ along the step edge. The occupation of the formerly empty Si $p$ orbital causes a $s p^{2}+p \rightarrow s p^{3}$ rehybridization and moves the respective $\mathrm{Si}$ atom out of the planar $s p^{2}$ configuration [20].

How likely is a local transition to such an electron-doped $(2,2,1)$ configuration? In order to answer this question we first calculate the total energy along the reaction path where-within the $1 \times 6$ surface unit cell-a single $\mathrm{Si}$ atom with an originally empty $\mathrm{db}$ is raised from the flat $s p^{2}$ coordination to the $s p^{3}$ configuration, see Fig. 2. Here the Au chain dimerization is fixed at its average value at $100 \mathrm{~K}$. The movement of the Si edge atom is accompanied by an $\mathrm{Au} \rightarrow \mathrm{Si}$ db charge transfer, see the Supplemental Material [28] for band structures. Along the reaction path a metastable structure appears that locally corresponds to the $(2,2,1)$ structure [14]. The energy barrier hindering such a local $(2,2,0) \rightarrow(2,2,1)$ transformation amounts to $58 \mathrm{meV}$, far above the thermal energy at $65 \mathrm{~K}$, i.e, about $6 \mathrm{meV}$. The energy barrier may be reduced at finite temperatures due to entropy effects [30]. In order to assess their magnitude, free-energy calculations were performed within the adiabatic approximation [31] based on surface vibrational modes determined in harmonic approximation by frozen-phonon calculations. As shown in Fig. 2, entropy effects indeed ease the $(2,2,0) \rightarrow(2,2,1)$ transition, but not sufficiently enough to explain a transition close to $65 \mathrm{~K}$.

In order to probe the possibility that a concerted movement involving a variety of atoms causes the $s p^{2}+p \rightarrow$ $s p^{3}$ rehybridization and the accompanying charge transfer, AIMD is performed. The time evolution (after equilibration) of the Si step edge atom vertical positions is shown in Fig. 3. Here it is discriminated between three configurations: $s p^{2}$ hybridized $\mathrm{Si}$ with an empty $p$ orbital $\mathrm{db}$, partially $s p^{3}$ hybridized Si hosting a single electron in its $\mathrm{db}$, and completely $s p^{3}$ hybridized $\mathrm{Si}$ with fully occupied $\mathrm{db}$. The $1 \times 6$ surface unit cell considered in the calculations is nearly entirely characterized by $(2,2,0)$ structures at $50 \mathrm{~K}$. In fact, there are only two very short occurrences of singly occupied $\mathrm{Si}$ dbs. These occurrences get more frequent and last longer at $100 \mathrm{~K}$. Also an emptying of the originally doubly occupied dbs is observed at this temperature. Still, the $(2,2,0)$ configuration is the most dominant structural motif at $100 \mathrm{~K}$. However, strong vertical vibrations of the Si step edge atoms occur. These vibrations get more pronounced for higher temperatures and quench the dominance of the $(2,2,0)$ structure. Increasing disorder is observed and singly occupied dbs occur frequently for
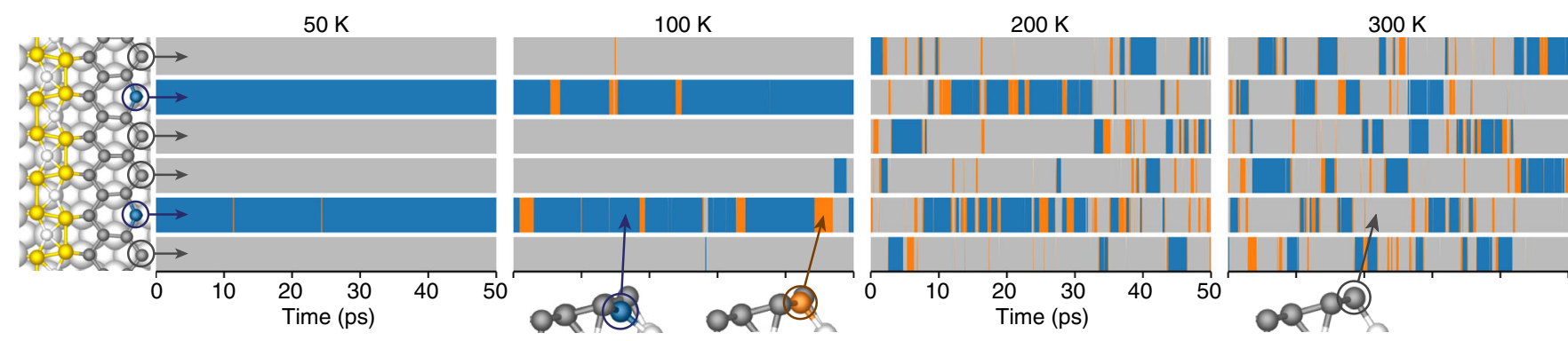

FIG. 3. Projection of the AIMD calculated Si step edge atom vertical positions on the heights corresponding to Si with empty $(q<0.5 e$, blue), singly occupied $(0.5 e \leq q \leq 1.5 e$, orange $)$, and doubly occupied $(q>1.5 e$, gray) dbs. 


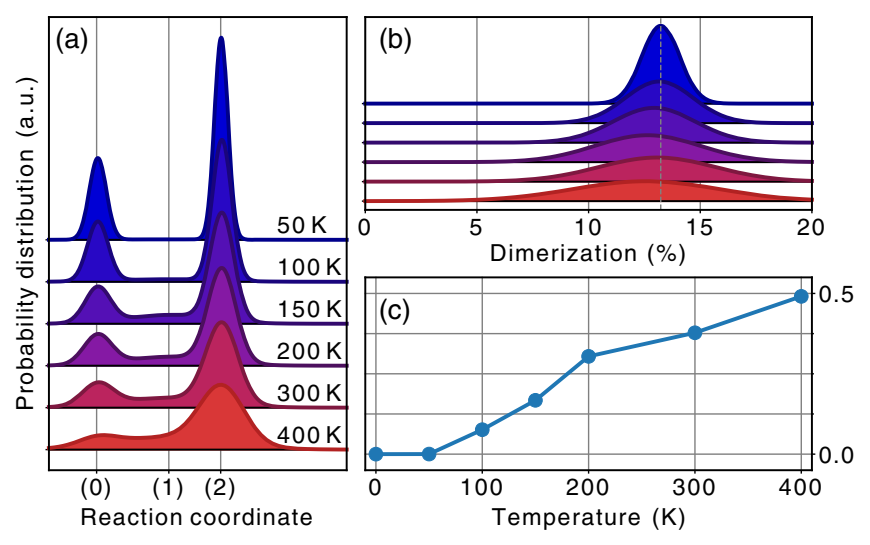

FIG. 4. (a) Distribution of the Si step edge atom vertical positions for various temperatures. Equilibrium positions of $\mathrm{Si}$ with empty (0), singly (1), and doubly (2) occupied dbs are indicated. (b) Temperature dependent distribution of $\mathrm{Au}$ chain dimerization. Color coding as shown left. (c) Probability of $(2,2,1)$ ordered surface domains derived from the dimerization distributions shown in (b), see text.

temperatures in excess of $200 \mathrm{~K}$. The AIMD results clearly show an order-disorder type phase transition.

The distribution function of the Si edge atom vertical positions in Fig. 4(a) provides additional details. At $50 \mathrm{~K}$ the distribution is bimodal, with cluster points for completely filled and empty $\mathrm{Si}$ dbs. A third, very weak accumulation point in between appears for $\mathrm{T}>50 \mathrm{~K}$. It corresponds to the occupation of the local energy minimum of the reaction path shown in Fig. 2, i.e., singly occupied dbs. For higher temperatures, the distribution gets broader and the Si vertical positions scatter broadly. This shows that the description of the phase transition in terms of solitons and antisolitons [32], i.e., binary occupancy shifts along the db wire, simplifies the actual surface dynamics.

Why does the order-disorder transition start at far lower temperatures than expected from the rehybridization barrier (black line in Fig. 2)? The answer is related to the electron chemical potential of the Au chain. A strongly dimerized chain has a stronger electron affinity than a weakly or undimerized chain [33]. Therefore, a reduced dimerization favors the $\mathrm{Au} \rightarrow$ Si charge transfer required for the rehybridization. In Fig. 2 it can be seen that a reduction of the dimerization from $12.8 \%$ (average value at $100 \mathrm{~K}$ ) to $10.0 \%$ reduces the $s p^{2}+p \rightarrow s p^{3}$ transition barrier (blue line) as strongly as doping the $1 \times 6$ unit cell with 0.4 electrons (red line). While the average dimerization is larger than $10.0 \%$ even at $400 \mathrm{~K}$, already for temperatures above $50 \mathrm{~K} \mathrm{Au}$ chain segments with $10.0 \%$ dimerization occur temporarily, cf. distribution in Fig. 4(b). With increasing temperature, even lower dimerization values occur and reduce locally the energy barrier for the $\mathrm{Si} s p^{2}+p \rightarrow s p^{3}$ rehybridization. Because of the nonlinear dependence of the reaction rate on the energy barrier, the rehybridization probability depends

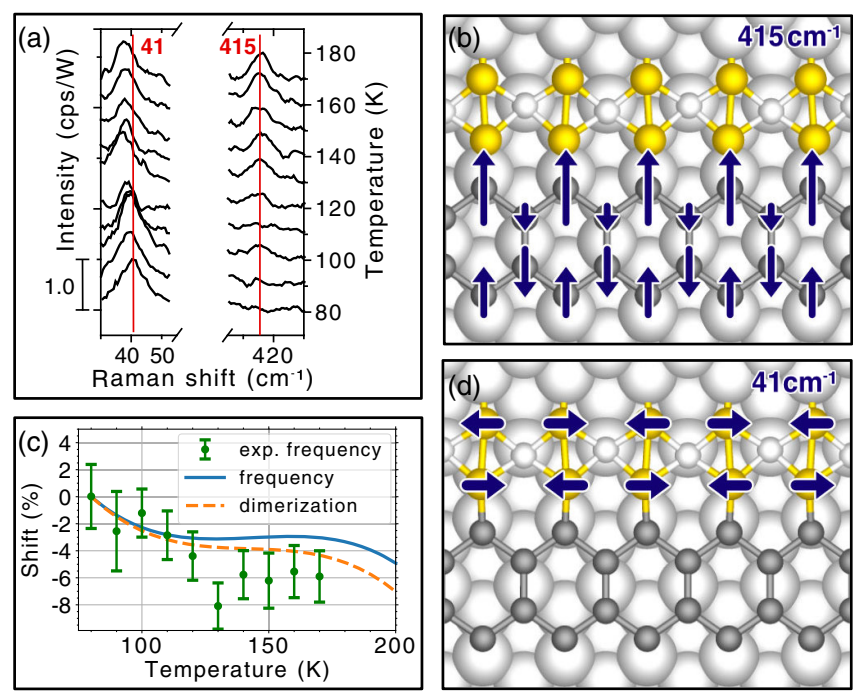

FIG. 5. (a) Temperature-dependent Raman spectra, focusing on a Raman mode located at the step edge at $415 \mathrm{~cm}^{-1}$ depicted in (b) and one located at the Au chain at $41 \mathrm{~cm}^{-1}$ depicted in (d). The measured frequency shift of the $41 \mathrm{~cm}^{-1}$ mode is in (c) compared with the calculated shift and the relative change in dimerization.

not only on the average dimerization, but on its entire distribution function. The probability for the occurrence of self-doped $(2,2,1)$ structures derived from the temperaturedependent dimerization distribution under the assumption of thermodynamic equilibrium between $(2,2,0)$ and $(2,2,1)$ surface domains is shown in Fig. 4(c). Its rise for temperatures above $50 \mathrm{~K}$ is - even quantitatively-consistent with the AIMD findings discussed above and explains the disorder along the Si step edge already at moderate temperatures.

Are these computational findings in agreement with the actual surface dynamics? First the temperature dependence of the surface Raman signatures is explored. Two signatures that are particularly strongly affected by temperature are shown in Fig. 5(a): There is a low-frequency phonon mode at around $41 \mathrm{~cm}^{-1}$ that softens considerably with rising temperature. A phonon mode at around $415 \mathrm{~cm}^{-1}$ appears slightly below $100 \mathrm{~K}$ and strongly gains intensity with rising temperature. Both signatures show a continuous rather than an abrupt change in energy or intensity, respectively. This supports the interpretation of the AIMD results in terms of an order-disorder transition.

Frozen-phonon calculations for $\mathrm{Si}(553)$ - $\mathrm{Au}$ surfaces allow for a microscopic interpretation of the Raman data. The calculations show the existence of a Au chain dimerization mode at $41 \mathrm{~cm}^{-1}$ for the $(2,2,0)$ ground state, see Fig. 5(d). Its thermal frequency shift-obtained by projecting the AIMD atomic velocities on the phonon eigenvector-is in close agreement with the low-energy Raman signature described above, see Fig. 5(c). The phonon-mode softening with rising temperature roughly 


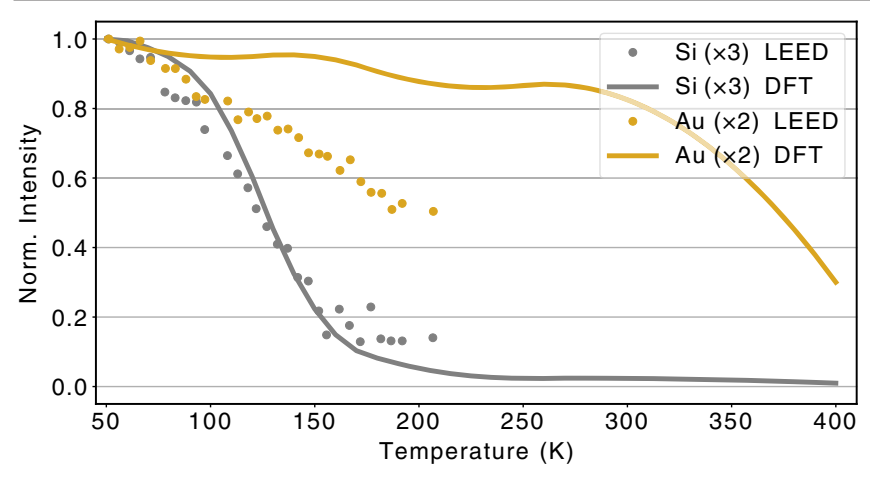

FIG. 6. Temperature-dependent $\times 3$ and $\times 2$ LEED spot intensities from Ref. [25] vs average structure factors including Debye-Waller effects obtained from the AIMD geometries.

parallels the reduction of the dimer strength, i.e., is caused by the reduction of the average $\mathrm{Au}-\mathrm{Au}$ bond strength. Because of its very low frequency, the Au chain mode is occupied already at a low temperature. It reduces temporarily the Au chain dimerization and thus lowers-as discussed above-the $s p^{2}+p \rightarrow s p^{3}$ activation energy.

Frozen-phonon calculations for the $(2,2,1)$ surface predict a transversal shear mode along the Si honeycomb chain with a frequency of $415 \mathrm{~cm}^{-1}$, see Fig. 5(b). Its frequency coincides with that of the high-temperature mode seen by Raman spectroscopy. This mode is replaced by localized $\mathrm{Si}$ vibrations for the $(2,2,0)$ structure, due to the stronger variation of the force constants along a step edge where $s p^{3}$ and $s p^{2}$ hybridized $\mathrm{Si}$ atoms alternate. This explains, why the measured mode disappears upon cooling the sample below $100 \mathrm{~K}$.

The calculated surface geometries are furthermore consistent with the evolution of the LEED data, see Fig. 6. Here the $\times 3$ and $\times 2$ LEED intensities measured in Ref. [25] are compared with the squared structure factors including Debye-Waller effects obtained from AIMD. Both the measured data and the calculations show a gradual loss of order starting below $100 \mathrm{~K}$, which first affects the $\times 3$ signatures and subsequently the $\times 2$ features, in agreement with earlier STM observations [22,23]. The quenching of the $\times 3$ signals is well reproduced by the calculated orderdisorder transition at the $\mathrm{Si}$ step edge, whereas the measured disappearance of the $\times 2$ intensities clearly precedes the calculated $\mathrm{Au}$ chain order-disorder transition. This is, however, to be expected: While the AIMD calculations find a broadening of the dimerization distribution, cf. Fig. 4(b), they cannot correctly account for disorder arising from dimerization phase shifts. The relatively small $1 \times 6$ unit cell leads to an overestimation of the defect or antidefect interaction that blueshifts the calculated critical temperature. Since the $\mathrm{Au}$ atoms are directly bonded, this effect is more relevant for the Au chain than for indirectly interacting step edge $\mathrm{Si}$.

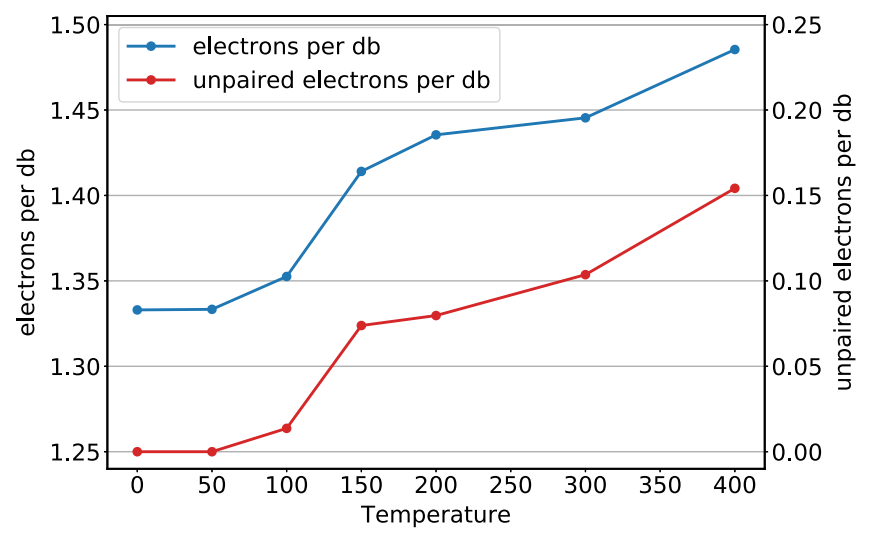

FIG. 7. Calculated average number of (unpaired) electrons per dangling bond.

Both the total number of electrons as well as the number of unpaired electrons in the $\mathrm{db}$ wires strongly increase for temperatures in excess of $50 \mathrm{~K}$, see Fig. 7. This reflects the increasing probability for $(2,2,0) \rightarrow(2,2,1)$ surface structure transitions, i.e., the temperature induced selfdoping of the surface. Similar to other activated atomic wire systems [34-36], the structural transition is accompanied by strong band structure modifications. As shown in the Supplemental Material [28], the weakly dispersing Si step edge states do not only shift in energy, they also hybridize with the Au chain states for thermally activated surface structures. This reduces drastically the effective masses characteristic for the Au bands [28]. The measured increase of the surface conductivity for temperatures above $65 \mathrm{~K} \mathrm{[25]} \mathrm{is} \mathrm{thus} \mathrm{probably} \mathrm{related} \mathrm{to} \mathrm{Si-modified} \mathrm{Au} \mathrm{states.}$ This interpretation is supported by the experimental finding that toward higher temperatures the surface conductivity decreases again, which is a hallmark of metallic transport [25].

To conclude, the present DFT calculations and Raman measurements do not only provide for the first time a consistent description of the experimentally observed twostage $\mathrm{Si}(553)$-Au surface phase transition, they also reveal a novel mechanism for the self-doping of db nanostructures: Thermally excited vibrations of the Au charge reservoir lead to transient changes of its electron affinity and thus facilitate electron doping at low temperatures. This mechanism can be expected to be relevant beyond the family of gold-stabilized Si surfaces, and to be conveniently tuned by modifying the species of the charge reservoir, the metal coverage, and the db density.

Financial support by DFG (FOR1700, SCHM1361/25, SCHM1361/26, TRR142 Project No. 231447078), Senatskanzlei Berlin, MKW NRW, BMBF, and EFRE (Project No. 1.8/07) is acknowledged. We thank the Paderborn Center for Parallel Computing $\left(\mathrm{PC}^{2}\right)$ and the Höchstleistungs-Rechenzentrum Stuttgart (HLRS) for grants of high-performance computer time. 
*christian.braun@upb.de

[1] On-Surface Atomic Wires and Logic Gates, edited by M. Kolmer and C. Joachim (Springer International Publishing AG, Berlin, 2017).

[2] S. R. Schofield, P. Studer, C. F. Hirjibehedin, N. J. Curson, G. Aeppli, and D. R. Bowler, Nat. Commun. 4, 1649 (2013).

[3] A. Kleshchonok, R. Gutierrez, C. Joachim, and G. Cuniberti, Sci. Rep. 5, 14136 (2015).

[4] K. Ambal, P. Rahe, A. Payne, J. Slinkman, C. C. Williams, and C. Boehme, Sci. Rep. 6, 18531 (2016).

[5] G. Grüner, Density Waves in Solids (Perseus Publishing, Cambridge, UK, 2000).

[6] T. Hitosugi, S. Heike, T. Onogi, T. Hashizume, S. Watanabe, Z.-Q. Li, K. Ohno, Y. Kawazoe, T. Hasegawa, and K. Kitazawa, Phys. Rev. Lett. 82, 4034 (1999).

[7] T. Frigge, B. Hafke, T. Witte, B. Krenzer, C. Streubühr, A. Samad Syed, V. Mikšić Trontl, I. Avigo, P. Zhou, M. Ligges et al., Nature (London) 544, 207 (2017).

[8] C. W. Nicholson, A. Lücke, W. G. Schmidt, M. Puppin, L. Rettig, R. Ernstorfer, and M. Wolf, Science 362, 821 (2018).

[9] J. Lahiri, Y. Lin, P. Bozkurt, I. I. Oleynik, and M. Batzill, Nat. Nanotechnol. 5, 326 (2010).

[10] A. Kleshchonok, R. Gutierrez, C. Joachim, and G. Cuniberti, Appl. Phys. Lett. 107, 203109 (2015).

[11] M. Engelund, N. Papior, P. Brandimarte, T. Frederiksen, A. Garcia-Lekue, and D. Sánchez-Portal, J. Phys. Chem. C 120, 20303 (2016).

[12] S. Bohloul, Q. Shi, R. A. Wolkow, and H. Guo, Nano Lett. 17, 322 (2017).

[13] F. Edler, I. Miccoli, J. P. Stöckmann, H. Pfnür, C. Braun, S. Neufeld, S. Sanna, W. G. Schmidt, and C. Tegenkamp, Phys. Rev. B 95, 125409 (2017).

[14] S. C. Erwin and F. J. Himpsel, Nat. Commun. 1, 58 (2010).

[15] J. Aulbach, S. C. Erwin, R. Claessen, and J. Schäfer, Nano Lett. 16, 2698 (2016).

[16] J. N. Crain, A. Kirakosian, K. N. Altmann, C. Bromberger, S. C. Erwin, J. L. McChesney, J. L. Lin, and F. J. Himpsel, Phys. Rev. Lett. 90, 176805 (2003).

[17] J. Aulbach, J. Schäfer, S. C. Erwin, S. Meyer, C. Loho, J. Settelein, and R. Claessen, Phys. Rev. Lett. 111, 137203 (2013).
[18] S. Polei, P. C. Snijders, S. C. Erwin, F. J. Himpsel, K.-H. Meiwes-Broer, and I. Barke, Phys. Rev. Lett. 111, 156801 (2013).

[19] M. Krawiec, Phys. Rev. B 81, 115436 (2010).

[20] C. Braun, U. Gerstmann, and W. G. Schmidt, Phys. Rev. B 98, 121402(R) (2018).

[21] J. Aulbach, S. C. Erwin, J. Kemmer, M. Bode, J. Schäfer, and R. Claessen, Phys. Rev. B 96, 081406(R) (2017).

[22] P. C. Snijders, S. Rogge, and H. H. Weitering, Phys. Rev. Lett. 96, 076801 (2006).

[23] J. R. Ahn, P. G. Kang, K. D. Ryang, and H. W. Yeom, Phys. Rev. Lett. 95, 196402 (2005).

[24] B. Hafke, T. Frigge, T. Witte, B. Krenzer, J. Aulbach, J. Schäfer, R. Claessen, S. C. Erwin, and M. Horn-von Hoegen, Phys. Rev. B 94, 161403(R) (2016).

[25] F. Edler, I. Miccoli, H. Pfnür, and C. Tegenkamp, Phys. Rev. B 100, 045419 (2019).

[26] G. Kresse and J. Furthmueller, Phys. Rev. B 54, 11169 (1996).

[27] J. P. Perdew, A. Ruzsinszky, G. I. Csonka, O. A. Vydrov, G. E. Scuseria, L. A. Constantin, X. Zhou, and K. Burke, Phys. Rev. Lett. 100, 136406 (2008).

[28] See Supplemental Material at http://link.aps.org/ supplemental/10.1103/PhysRevLett.124.146802 for calculated band structures and effective electron masses, which includes Ref. [29].

[29] P. V. C. Medeiros, S. Stafström, and J. Björk, Phys. Rev. B 89, 041407(R) (2014).

[30] S. Wippermann and W. G. Schmidt, Phys. Rev. Lett. 105, 126102 (2010).

[31] M. Valtiner, M. Todorova, G. Grundmeier, and J. Neugebauer, Phys. Rev. Lett. 103, 065502 (2009).

[32] B. Hafke, C. Brand, T. Witte, B. Sothmann, M. Horn-von Hoegen, and S. C. Erwin, Phys. Rev. Lett. 124, 016102 (2020)

[33] C. Hogan, E. Speiser, S. Chandola, S. Suchkova, J. Aulbach, J. Schäfer, S. Meyer, R. Claessen, and N. Esser, Phys. Rev. Lett. 120, 166801 (2018).

[34] C. González, F. Flores, and J. Ortega, Phys. Rev. Lett. 96, 136101 (2006).

[35] D. Sánchez-Portal, S. Riikonen, and R. M. Martin, Phys. Rev. Lett. 93, 146803 (2004).

[36] S. Riikonen and D. Sánchez-Portal, Phys. Rev. B 76, 035410 (2007). 